\title{
Synergistic effects of intracoronary infusion of autologous bone marrow-derived mesenchymal stem cells and revascularization procedure on improvement of cardiac function in patients with severe ischemic cardiomyopathy
}

\author{
Sze Piaw Chin ${ }^{1,2}$, Oteh Maskon ${ }^{3}$, Chiang Soo Tan ${ }^{4}$, John E. Anderson ${ }^{4}$, Chee Yin Wong ${ }^{1}$, \\ Hamat Hamdi Che Hassan ${ }^{3}$, Chee Ken Choor ${ }^{3}$, S. Abdul Wahid Fadilah ${ }^{5}$, Soon Keng Cheong ${ }^{1,6}$ \\ ${ }^{1}$ Cytopeutics, Selangor, Malaysia; ${ }^{2} \mathrm{CMH}$ Specialist Hospital, Negeri Sembilan, Malaysia; ${ }^{3}$ Cardiology Unit, Department of Medicine, Universiti \\ Kebangsaan Malaysia Medical Centre, Kuala Lumpur, Malaysia; ${ }^{4}$ Adventist Heart Centre, Penang Adventist Hospital, Penang, Malaysia; ${ }^{5}$ Cell \\ Therapy Centre, Faculty of Medicine, Universiti Kebangsaan Malaysia Medical Centre, Kuala Lumpur, Malaysia; ${ }^{6}$ Faculty of Medicine and Health \\ Sciences, Tunku Abdul Rahman University, Selangor, Malaysia \\ Contributions: (I) Conception and design: SP Chin, O Maskon, SAW Fadilah, SK Cheong; (II) Administrative support: SP Chin, O Maskon, SAW \\ Fadilah, SK Cheong; (III) Provision of study materials or patients: O Maskon; CS Tan, JE Anderson, HHC Hassan, CK Choor; (IV) Collection and \\ assembly of data: SP Chin, CY Wong; (V) Data analysis and interpretation: SP Chin, O Maskon, CY Wong; (VI) Manuscript writing: All authors; (VII) \\ Final approval of manuscript: All authors. \\ Correspondence to: Sze Piaw Chin. Cytopeutics, Suite 2-3, 2nd Floor, Bio-X Centre, Persiaran Cyberpoint Selatan, Cyber 8, 63000 Cyberjaya, \\ Selangor, Malaysia. Email: chin.sze.piaw@gmail.com.
}

\begin{abstract}
Background: Ischemic cardiomyopathy (ICM) is a leading cause of cardiovascular mortality worldwide. It is defined as abnormal enlargement of the left ventricular (LV) cavity with poor LV function due to coronary artery disease. Currently available established treatments are palliative whereby blood supply is recovered to ischemic regions but fails to regenerate heart tissues. Mesenchymal stem cells (MSCs) offer a promising treatment for ICM given their regenerative and multipotent characteristics. This study aims to investigate the effect of MSCs infusion with concurrent revascularization in patients with severe ICM compared to receiving only revascularization procedure or MSCs infusion.

Methods: Twenty-seven patients with history of anterior myocardial infarction (MI) and baseline left ventricular ejection fraction (LVEF) of less than $35 \%$ were recruited into this study. Patients who are eligible for revascularization were grouped into group A (MSCs infusion with concurrent revascularization) or group B (revascularization only) while patients who were not eligible for revascularization were allocated in group $\mathrm{C}$ to receive intracoronary MSCs infusion. LV function was measured using echocardiography.

Results: Patients who received MSCs infusion (either with or without revascularization) demonstrated significant LVEF improvements at 3, 6 and 12 months post-infusion when compared to baseline LVEF within its own group. When comparing the groups, the magnitude of change in LVEF from baseline for third visits i.e., 12 months post-infusion was significant for patients who received MSCs infusion plus concurrent revascularization in comparison to patients who only had the revascularization procedure.

Conclusions: MSCs infusion significantly improves LV function in ICM patients. MSCs infusion plus concurrent revascularization procedure worked synergistically to improve cardiac function in patients with severe ICM.
\end{abstract}

Keywords: Intracoronary; ischemic cardiomyopathy (ICM); mesenchymal stem cell (MSC); revascularization

Received: 12 June 2020; Accepted: 18 December 2020; Published: 22 January 2021.

doi: $10.21037 /$ sci-2020-026

View this article at: http://dx.doi.org/10.21037/sci-2020-026 


\section{Introduction}

The latest Global Health Estimates (GHE) by World Health Organization (WHO) reported ischemic heart disease as the leading cause of death worldwide, accounting for 7.35 million deaths $(13.2 \%$ of total death) in year 2012 (1). Ischemic cardiomyopathy (ICM) is a condition defined by the weakening of the heart to circulate blood effectively due to coronary artery disease and the narrowing or hardening of the arteries. This condition can result in the loss of cardiomyocytes, scarring and reduced ventricular contractility that will lead to heart failure, and finally death (2).

Currently available established treatments for ICM are limited to revascularization strategies such as percutaneous transluminal coronary angioplasty (PTCA) and coronary artery bypass graft (CABG) operations. Implantation of pacemaker or defibrillator is considered for patients with cardiac dyssynchrony or risk of ventricular arrhythmias. For patients with end-stage cardiomyopathy, heart transplantation is the final option. Despite the treatment advances, the only strategy that addresses the loss of cardiomyocytes is heart transplantation. However, this method is severely limited due to the scarce availability of organ, and graft rejection (3). Revascularization is an effective method in rejuvenating viable and dormant cardiomyocytes but it is unable to restore function of the damaged cardiomyocytes (4).

Therefore, the use of mesenchymal stem cells (MSCs) serves as a promising therapeutic strategy for the treatment of ICM given that MSCs have the potential to enter cell cycle and differentiate into cardiomyocytes to replace the infarcted cardiomyocytes (5). Studies have shown beneficial effects of MSCs for treating myocardial infarction (MI) through intracoronary and intravenous administration with improvement of left ventricular ejection fraction (LVEF), left ventricular (LV) remodelling and reduction of infarct size (6). Infusion of MSCs in acute MI has resulted in the reduction of heart failure, rehospitalization due to cardiac complications, and cardiac hypertrophy too (7).

Our previous studies have demonstrated that autologous MSCs stored by cryopreservation is safe and feasible for transportation without affecting the cell viability and risk of contamination (8). With assurance that the cells are well-maintained before infusion, we moved on to prove that autologous MSCs treatment via intracoronary or intramyocardial administration is effective and safe for treatment of chronic severe dilated cardiomyopathy with improvement of $\mathrm{LV}$ function and parameters, and the resolution of full thickness scarring (9). In this study, we would like investigate the effect of autologous MSCs infusion for severe ICM by comparing three different modes of treatment; MSCs intracoronary infusion only, revascularization only and the combination of MSCs intracoronary infusion with revascularization.

\section{Methods}

\section{Study design and patient selection}

Patients aged between 35 and 70 years were recruited from participating cardiology clinics. Patients were considered for participation in the study according to criteria: (I) diagnosed to have ICM with history of previous anterior MI, (II) LVEF of less than 35\% without any significant improvement within the past 6 months, and (III) symptomatic heart failure in New York Heart Association (NYHA) functional class II to IV with evidence of failure despite optimal heart disease medication or inability to attain maximal therapy due to side effects.

Exclusion criteria include patients with: (I) heart failure due to other causes such as idiopathic, infective or metabolic cardiomyopathy, valvular heart disease and pericardial disease, (II) contradictions to bone marrow aspiration, (III) contradiction to coronary contrast angiography and angioplasty, (IV) infectious diseases including hepatitis B, hepatitis $\mathrm{C}$ and HIV, (V) history of neoplasia and primary hematological disease, (VI) renal impairment (creatinine clearance less than $30 \mathrm{~mL} / \mathrm{min}$ ), and (VII) liver impairment (liver transaminase level at four times higher than the optimal value).

Based on these inclusion and exclusion criteria, 27 patients who had anterior MI previously and baseline cardiac function (LVEF) less than 35\% were recruited (Figure 1). Patients eligible for revascularization were divided into group A (MSCs infusion concurrent with revascularization) and B (revascularization only). Patients who were not suitable for revascularization were set apart in group C for intracoronary infusion of MSCs. Patients not suitable for revascularization are those with diffuse small vessel disease or chronic total occlusion with collateral flow.

This is a multicentre study and was conducted as a controlled open label trial in accordance with Malaysian Guidelines for Stem Cell Research and Therapy (Ministry of Health Malaysia, 2009). The study was conducted in accordance with the Declaration of Helsinki (as revised in 2013). Study was approved by the Research Committee 


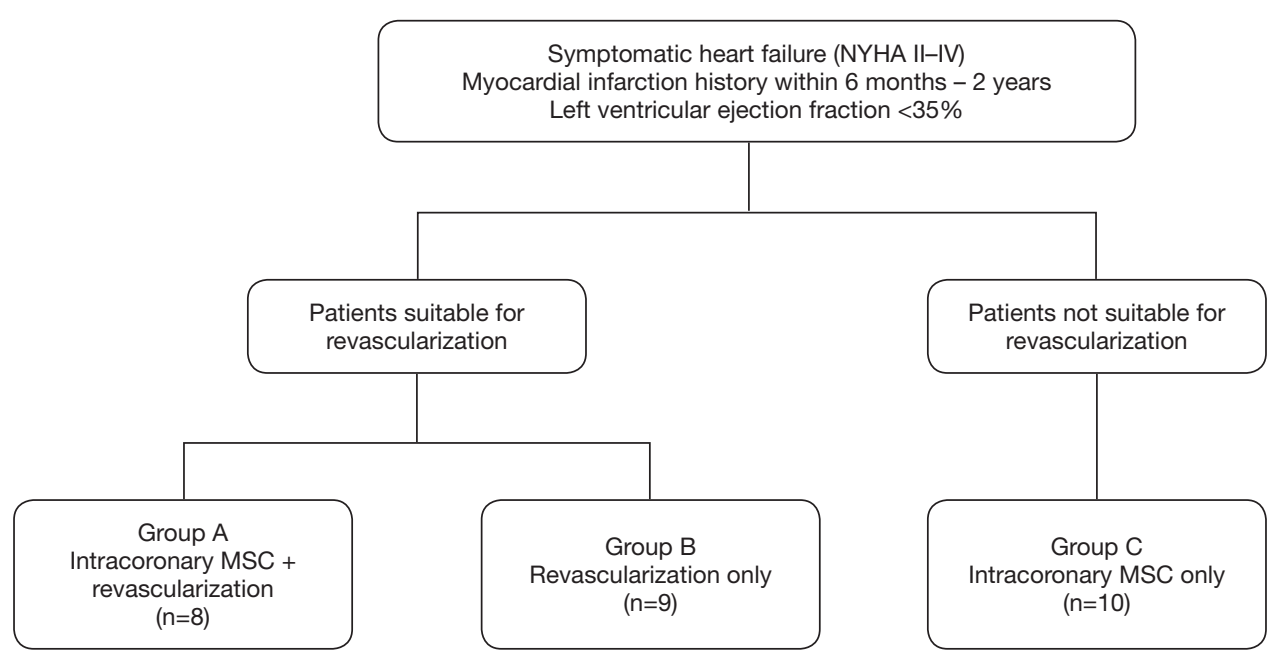

Figure 1 Distribution of recruited patients in this study. Twenty-seven patients were allocated into three different groups. Eight patients were allocated to group A for intracoronary MSCs infusion with concurrent revascularization; nine patients were assigned to group B for revascularization procedure only while ten patients who were not amenable to revascularization were allocated in group $\mathrm{C}$ for intracoronary infusion of MSCs only. MSC, mesenchymal stem cell; NYHA, New York Heart Association.

of National University of Malaysia Medical Centre with oversight provided by the Malaysian Medical Research and Ethics Committee (Ref. No. ERGS/1/2011/SKK/ $\mathrm{UKM} / 02 / 71$ ) and informed consent was taken from all individual participants. The study has registered under ClinicalTrial.gov (NCT 01720888).

\section{MSCs culture and storage}

These methods have been previously described (8-10). Briefly, $50 \mathrm{~mL}$ of bone marrow aspirates (BMA) were obtained from the iliac crest of each patient and processed within 24 hours. MSCs were isolated from BMA using Ficoll-Paque density gradient centrifugation and adhered to the surface of culture flask. MSCs were cultured in Dulbecco's modified Eagle's medium with low glucose (Gibco) supplemented with 10\% autologous serum, $100 \mathrm{U} / \mathrm{mL}$ penicillin, $100 \mathrm{mg} / \mathrm{mL}$ streptomycin, $250 \mathrm{ng} / \mathrm{mL}$ amphotericin B and $2 \mathrm{mM}$ GlutaMAX (Gibco). MSCs were maintained in a $37{ }^{\circ} \mathrm{C}$ humidified incubator with $5 \% \mathrm{CO}_{2}$ and $95 \%$ air.

After 3 days, cells in suspension were discarded and culture medium was replaced every 3-4 days until confluent. MSCs adhered to the flask were harvested using TrypLE Select (Gibco) and seeded into new culture flask for population expansion. Upon achieving the required number of cells, MSCs were harvested and cryopreserved in $90 \%$ autologous plasma and 10\% DMSO. MSCs were characterized by immunophenotyping and checked for their differentiation abilities according to the method described previously (11). The cells were also checked for sterility from microbial and fungal contaminations.

For MSCs infusion, cryopreserved MSCs were transported to the medical centre in a cryoshipper. MSCs were thawed before resuspended in $10 \mathrm{~mL}$ sterile $0.9 \%$ normal saline for intracoronary infusion.

\section{Intracoronary infusion of autologous MSCs}

Intracoronary infusion was performed according to the standard procedure as practiced in current interventional cardiology. Arterial puncture was done and arterial sheath was then inserted to the punctured artery.

An angioplasty guide catheter was inserted to the infarct coronary artery. Then, an over the wire balloon catheter (oversized by $0.5 \mathrm{~mm}$ ) was advanced by a standard guidewire threaded in the infract artery. At a ratio of $1: 1$, contrast material was added to a syringe containing MSCs suspension. Guidewire was removed and replaced by the MSCs-contrast syringe syringe. The balloon was inflated at 2-3 atm for 1-2 minutes to block the blood flow.

Next, 1-2 mL of MSCs suspension were infused over 1 minute through the guidewire and flushed with $1 \mathrm{~mL}$ of heparinized saline. This step was repeated two to three 
Table 1 Patients demographic data, echocardiographic parameters and comorbidity

\begin{tabular}{|c|c|c|c|c|c|}
\hline \multirow{2}{*}{ Parameters } & \multirow{2}{*}{ Total (mean \pm SD) } & \multicolumn{3}{|c|}{ Group (mean \pm SD) } & \multirow{2}{*}{$P$ value } \\
\hline & & A & $\mathrm{B}$ & $\mathrm{C}$ & \\
\hline No. of patients $(n)$ & 27 & 8 & 9 & 10 & \\
\hline Age (years) & $57.4 \pm 10.4$ & $53.3 \pm 12.9$ & $59.1 \pm 8.7$ & $59.1 \pm 9.7$ & 0.42 \\
\hline Male (n) & 26 & 8 & 9 & 9 & \\
\hline Female (n) & 1 & 0 & 0 & 1 & \\
\hline Smoker (n) & 9 & 5 & 0 & 4 & \\
\hline Diabetes mellitus ( $n$ ) & 13 & 4 & 4 & 5 & \\
\hline Hypertension (n) & 15 & 4 & 6 & 5 & \\
\hline Ischemic heart disease (n) & 15 & 4 & 6 & 5 & \\
\hline Hyperlipidaemia (n) & 21 & 8 & 6 & 7 & \\
\hline LVEF baseline (\%) & $29.3 \pm 6.4$ & $27.5 \pm 5.6$ & $28.0 \pm 8.3$ & $32.0 \pm 4.5$ & 0.26 \\
\hline LVEDD baseline $(\mathrm{cm})$ & $6.4 \pm 0.7$ & $6.8 \pm 0.7$ & $6.0 \pm 0.3$ & $6.5 \pm 0.7$ & 0.07 \\
\hline IVSD baseline (cm) & $1.0 \pm 0.2$ & $1.2 \pm 0.3$ & $1.0 \pm 0.2$ & $1.0 \pm 0.3$ & 0.22 \\
\hline
\end{tabular}

$P$ value $<0.05$ indicates statistical significant difference across three different groups of patients. SD, standard deviation; LVEF, left ventricular ejection fraction; LVEDD, left ventricular end diastolic diameter; IVSD, interventricular septal thickness at diastole.

times while the balloon was allowed to deflate between each infusion until all $10 \mathrm{~mL}$ of MSCs were infused. Two to three million MSCs $/ \mathrm{kg}$ were infused to the patients based on weight. Coronary angiogram was performed at the end of the infusion to ensure vessel patency and no other complications.

\section{Patients follow up and monitoring}

Patients who have been administered with autologous MSCs were observed in the coronary care unit for a minimum period of 24 hours to monitor for any ventricular arrhythmias using telemetry. Patients were required to return to the medical centre after 6 weeks, 3 months, 6 months and 12 months for follow up assessment. LV function and parameters such as, LVEF, left ventricular end diastolic diameter (LVEDD), and interventricular septal thickness at diastole (IVSD) were evaluated by echocardiography during each follow up.

\section{Statistical analysis}

Statistical analysis were performed using Microsoft Excel and SPSS version 14.0. Means of the values were summarized as mean \pm standard deviation (mean $\pm \mathrm{SD}$ ). Magnitude of change in LVEF from baseline $(\triangle \mathrm{LVEF})$ was calculated as follows:

$$
\frac{\text { Current LVEF - Baseline LVEF }}{\text { Baseline LVEF }} \times 100 \%
$$

The multiple comparisons of means of parameters for different time points were conducted using oneway ANOVA. T-tests were used for comparison between different groups. In this study, the significance level is set at $\mathrm{P}<0.05$ or unless stated otherwise.

\section{Results}

\section{Patient baseline clinical data and echocardiograph ventricular parameters}

In this study, there were 26 male patients and 1 female patient. Fifteen out of the 27 patients had ischemic heart disease and co-morbid hypertension while there were 21 patients with hyperlipidaemia. Nine of the patients are smokers or ex-smokers. Thirteen patients in this study had co-morbid diabetes mellitus. The average of the LVEF percentage at baseline is $29.3 \% \pm 6.4 \%$ for all patients. The total LVEDD baseline mean was $6.4 \pm 0.7 \mathrm{~cm}$. The mean value of IVSD baseline for the patients was recorded at $1.0 \pm 0.2 \mathrm{~cm}$ (Table 1). 


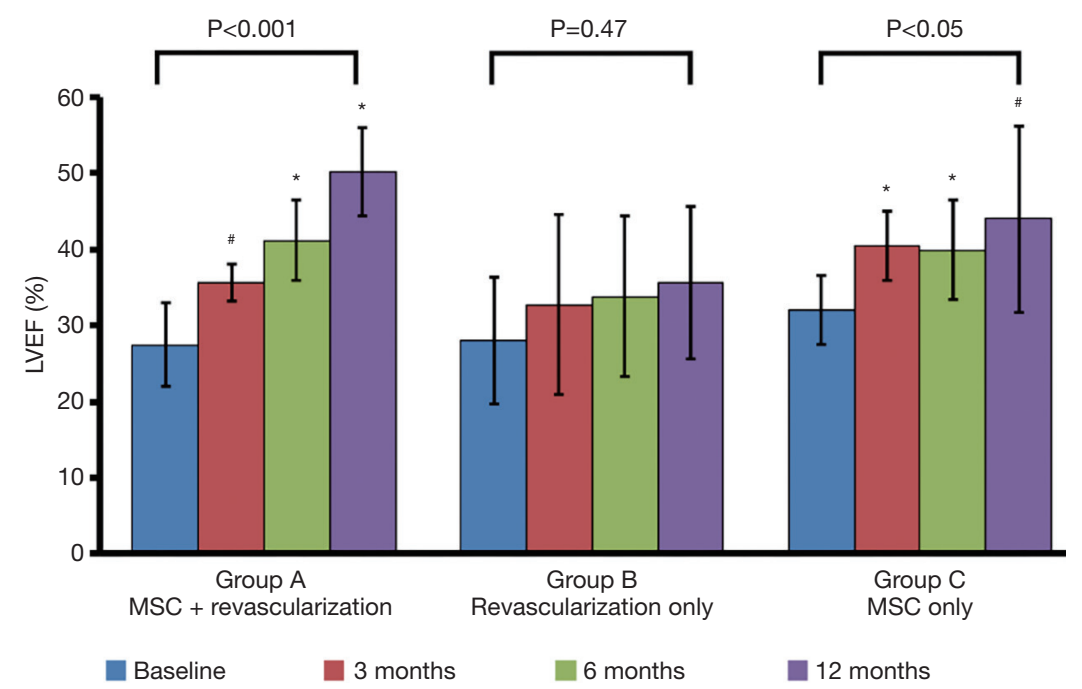

Figure 2 Comparison of mean of LVEF within groups at baseline, 3, 6 and 12 months visit. For patients in group A and C, LVEF showed significant improvement for all post-treatment visits when comparing to baseline. Significant values: ${ }^{\#}, \mathrm{P}<0.05$; *, P<0.01. LVEF, left ventricular ejection fraction.

\section{Significant improvement of LVEF after MSCs infusion}

LVEF measurements were obtained using echocardiogram (echo) at 3, 6 and 12 months post treatment and compared to baseline LVEF for patients in group A, B and C (Figure 2). There were significant LVEF improvement for patients in group A $(35.6 \pm 2.4,41.2 \pm 5.2$, and $50.2 \pm 5.8)$ at 3, 6 and 12 months post-MSCs infusion concurrent with revascularization. Patients who received MSCs infusion only also showed significant LVEF improvement at the 3,6 and 12 months post-treatment visits $(32.0 \% \pm 4.5 \%$, $40.5 \% \pm 4.5 \%$, and $39.9 \% \pm 6.6 \%$, respectively). For patients in group B, there were no significant changes in LVEF after revascularization when compared to baseline LVEF.

Mean of LVEF percentages for the 3 visits when compared with one-way ANOVA showed significant difference in group A and $\mathrm{C}$ up to 12 months of follow up $(\mathrm{P}<0.001$ and $\mathrm{P}<0.05$ respectively). However, there is no significant difference of LVEF in group B (revascularization only) in this statistic analysis (Table 2).

\section{Magnitude of change of LVEF in comparison between three groups}

The $\triangle \mathrm{LVEF}$ in third visit (12 months post-treatment) was significant for patients with MSCs infusion concurrent with revascularization (group A) when compared with patients who underwent revascularization procedure only (group B)
(Figure 3). However, there was no statistically significant difference between patients who received revascularization only (group A) and MSCs infusion only (group C).

\section{Discussion}

Usage of MSCs has been increasingly investigated for therapeutic purpose. The present study was designed to assess the safety and efficacy of the autologous MSCs infusion with concurrent revascularization for severe ICM in comparison to receiving autologous MSCs infusion alone or revascularization procedure only. Our findings suggest that the usage of MSCs infusion with concurrent revascularization is beneficial to patients with severe ICM which will provide important evidence for the use of this method in clinical setting.

Further on that, we have proven that intracoronary injection of autologous MSCs in patients with severe ICM was well-tolerated. No proarrythmia, calcification or tumor formation was observed in patients who underwent MSCs infusion. The infusion of MSCs was safe and was not associated with any kind of adverse effects. This finding is in line with the meta-analysis done by de Jong et al. on clinical trials of intracoronary stem cell infusion for MI which reported that trials have repeatedly proven that stem cell therapy is safe and feasible, and intracoronary stem cell infusion did not cause any adverse events (12). 
Table $2 \mathrm{LV}$ parameter measured by echocardiography for baseline and all three visits

\begin{tabular}{|c|c|c|c|c|c|c|}
\hline \multirow{2}{*}{ Parameters } & \multirow{2}{*}{ Group } & \multicolumn{4}{|c|}{ Visit $($ mean $\pm S D)$} & \multirow{2}{*}{$P$ value } \\
\hline & & Baseline & 3 months & 6 months & 12 months & \\
\hline \multirow[t]{3}{*}{ LVEF (\%) } & A & $27.5 \pm 5.6$ & $35.6 \pm 2.4$ & $41.2 \pm 5.2$ & $50.2 \pm 5.8$ & $<0.001$ \\
\hline & $\mathrm{B}$ & $28.0 \pm 8.3$ & $32.8 \pm 11.9$ & $33.8 \pm 10.5$ & $35.6 \pm 10.1$ & 0.47 \\
\hline & C & $32.0 \pm 4.5$ & $40.5 \pm 4.5$ & $39.9 \pm 6.6$ & $44.0 \pm 12.3$ & 0.02 \\
\hline \multirow[t]{3}{*}{ LVEDD $(\mathrm{cm})$} & A & $6.8 \pm 0.6$ & $6.5 \pm 0.8$ & $6.6 \pm 0.9$ & $6.3 \pm 1.0$ & 0.79 \\
\hline & B & $6.0 \pm 0.3$ & $5.7 \pm 0.4$ & $5.8 \pm 0.8$ & $5.8 \pm 0.4$ & 0.71 \\
\hline & C & $6.5 \pm 0.7$ & $6.2 \pm 1.3$ & $5.6 \pm 1.0$ & $6.2 \pm 0.8$ & 0.51 \\
\hline \multirow[t]{3}{*}{ IVSD (cm) } & A & $1.2 \pm 0.3$ & $1.2 \pm 0.2$ & $1.2 \pm 0.4$ & $1.1 \pm 0.0$ & 0.88 \\
\hline & $\mathrm{B}$ & $1.0 \pm 0.2$ & $1.0 \pm 0.2$ & $0.9 \pm 0.1$ & $1.0 \pm 0.1$ & 1.00 \\
\hline & $\mathrm{C}$ & $1.0 \pm 0.3$ & $1.1 \pm 0.3$ & $1.2 \pm 0.3$ & $1.2 \pm 0.2$ & 0.55 \\
\hline
\end{tabular}

$P$ value $<0.05$ indicates statistical significant difference across the baseline and three visits. LV, left ventricular; SD, standard deviation; LVEF, left ventricular ejection fraction; LVEDD, left ventricular end diastolic diameter; IVSD, interventricular septal thickness at diastole.

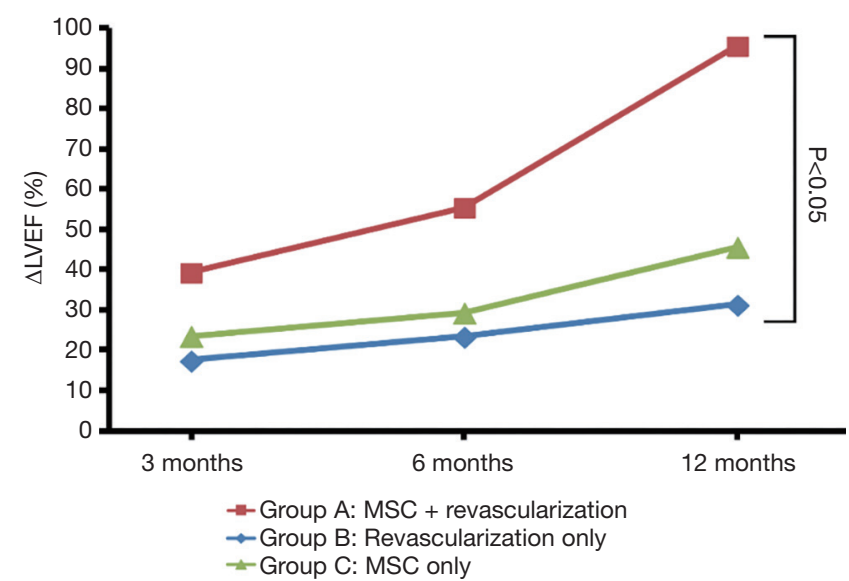

Figure 3 Comparison between three groups for the magnitude of change of LVEF compared to baseline during 3, 6 and 12 months visits. Magnitude of change is significant at the 12 months posttreatment visit when comparing between group A and B. LVEF, left ventricular ejection fraction; MSC, mesenchymal stem cell.

Our results also revealed that the administration of MSCs in the patients improved their LVEF. The effect is more eminent when combined with revascularization procedure demonstrating evidence that these two procedures can work synergistically. Some patients did not significantly benefit from revascularization procedure alone because the myocardium is not recovered by this process due to the presence of non-viable cardiomyocytes (13). The administration of MSCs further improves cardiac functions for patients when revascularization procedure fails to show significant improvements. This observation is shown by the evident improvement in the LVEF at 12 months after MSCs infusion in group A patients. The delayed benefit of MSCs infusion which was apparent after 12 months is consistent with the understanding of the principles of regenerative medicine. The delayed effect may be due to low engraftment of MSCs within the targeted sites following intracoronary infusion. Optimization should be carried out to improve the engraftment and homing of MSCs within the targeted sites. Our results suggest that adequate revascularization is probably essential to successful engraftment.

Infusion of MSCs may have contributed to the formation of cardiomyocytes that subsequently form renewed myocardium resulting in the improvement of the cardiac function. It has been shown that stem cells including embryonic stem cells, cardiac stem cells and induced pluripotent stem cells can differentiate into cardiomyocytes upon transplantation. This process is important to initiate angiogenesis, revive damaged heart muscle, and reduce scar expansion (14). It has also been demonstrated that adult MSCs can differentiate into cardiomyocytes in vitro and in vivo through an appropriate induction process $(15,16)$.

Studies have reported that stem cell treatment for heart failure is highly dependent on the mechanisms related to paracrine effect of stem cell which promote angiogenesis, and prevent cardiomyocytes apoptosis (17). Recent evidence has proven that MSCs-derived miRNA- 
bearing exosomes were internalized into cardiomyocytes to improve angiogenecity, anti-apoptotic features, and antiinflammatory activities (18). Increase angiogenesis and blood supply to ischemic areas improves revascularization of the hibernating myocardium (19).

Hibernating cardiomyocytes are prone to undergo necrosis or apoptosis that leads to heart failure. MSCs may induce the secretion of cytokines and growth factors that reduce the cell death of cardiomyocytes and facilitate vascular regeneration $(17,20)$. Hence, the significant improvement in the LVEF of group A patients at 12 months post-MSCs infusion can be due to the late effect of gradual recovery process of hibernating myocardium and regeneration which subsequently provide adequate vascular supply to the patients. It has been reported that bone marrow derived stromal cells implanted in the infarcted myocardium lose their haemopoietic properties and acquired cardiogenicity to form functional cardiomyocytes in vivo (21). Besides, bone marrow derived stem cells administration can reduce the activities of circulating autoantibodies mediating the tolerability of autoreactive lymphocytes during the pathogenesis of dilated cardiomyopathy (22). MSCs are able to impair the cytotoxic effect of T lymphocytes, inhibit differentiation of $\mathrm{B}$ cells and inhibit the immunostimulatory activity of dendritic cells (23). These observation demonstrated the immunomodulatory properties of MSCs as an early effect of MSCs in vivo or upon infusion.

\section{Limitation of the study}

The limitations of this study include the low number of recruited patients. The small number of patients recruited might contribute to the limited conclusions for the measurements done in this study. The stringent exclusion criterion is the main contributor to this limitation where patients eligible for other treatment options and would likely to benefit from revascularization alone were excluded. Although there is a limitation in sample size, the groups were very well-matched at the baseline which reduced potential bias in this study. One of the limitations in this study which is also a limitation in most of the clinical trials related to MSCs infusion is the inability to discern the fate of autologous MSCs in the body after infusion. This is the reason that no markers have been used in this study, and no modifications have been done to the infused MSCs. Therefore, MSCs cannot be detectable after infusion. Besides, placebo treatment was not included in this study which might introduce potential bias. Hence, we minimized the bias that could be potentially caused by this factor by assigning independent and blinded observers for echocardiographic evaluation during the follow up visits.

\section{Conclusions}

In summary, we would like to conclude that this study has provided clinical evidence that the usage of autologous MSCs is safe and effective for the treatment of severe ICM. Intracoronary injection of autologous MSCs at a suitable dose elicits improvement in the cardiac function in terms of LV function specifically in patients who have concurrent revascularization. The magnitude of LVEF changes was significant for patients with MSCs infusion and revascularization in comparison with patients who underwent revascularization procedure only.

\section{Acknowledgments}

Funding: The study was jointly sponsored by Cytopeutics Sdn. Bhd. and the Ministry of Higher Education Malaysia grant under project (ERGS/1/2011/SKK/UKM/02/71).

\section{Footnote}

Conflicts of Interest: All authors have completed the ICMJE uniform disclosure form (available at http://dx.doi. org/10.21037/sci-2020-026). Dr. SPC, Dr. CYW, and Dr. SKC report in addition, they have a patent MY-171690-A issued and affiliated with Cytopeutics Sdn. Bhd.; a joint sponsor and played roles in this study as declared under the "Contributions" section of the manuscript. Dr. OM, Dr. $\mathrm{HHCH}$, Dr. CKC, and Dr. AWF report grants from the Ministry of Higher Education Malaysia, during the conduct of the study. The other authors have no conflicts of interest to declare.

Ethical Statement: The authors are accountable for all aspects of the work in ensuring that questions related to the accuracy or integrity of any part of the work are appropriately investigated and resolved. This is a multicentre study and was conducted as a controlled open label trial in accordance with Malaysian Guidelines for Stem Cell Research and Therapy (Ministry of Health Malaysia, 2009). The study was conducted in accordance with the Declaration of Helsinki (as revised in 2013). Study 
was approved by the Research Committee of the National University of Malaysia Medical Centre with oversight provided by the Malaysian Medical Research and Ethics Committee (Ref. No. ERGS/1/2011/SKK/UKM/02/71) and informed consent was taken from all individual participants. The study has registered under ClinicalTrial. gov (NCT 01720888).

Open Access Statement: This is an Open Access article distributed in accordance with the Creative Commons Attribution-NonCommercial-NoDerivs 4.0 International License (CC BY-NC-ND 4.0), which permits the noncommercial replication and distribution of the article with the strict proviso that no changes or edits are made and the original work is properly cited (including links to both the formal publication through the relevant DOI and the license). See: https://creativecommons.org/licenses/by-nc-nd/4.0/.

\section{References}

1. World Health Organization. Global Health Estimates 2015 summary tables: global deaths by cause, age and sex, 2000-2015. Geneva: World Health Organization, 2016. Available online: http://www.who.int/entity/ healthinfo/global_burden_disease/GHE2015_Deaths_ Global_2000_2015.xls?ua=1

2. Nabel EG, Braunwald E. A tale of coronary artery disease and myocardial infarction. N Engl J Med 2012;366:54-63.

3. Zimmermann WH, Didié M, Döker S, et al. Heart muscle engineering: an update on cardiac muscle replacement therapy. Cardiovasc Res 2006;71:419-29.

4. Abbate A, Biondi-Zoccai GGL, Agostoni P, et al. Recurrent angina after coronary revascularization: a clinical challenge. Eur Heart J 2007;28:1057-65.

5. Pittenger MF, Martin BJ. Mesenchymal stem cells and their potential as cardiac therapeutics. Circ Res 2004;95:9-20.

6. Wollert KC, Drexler H. Mesenchymal stem cells for myocardial infarction. Circulation 2005;112:151-3.

7. Telukuntla KS, Suncion VY, Schulman IH, et al. The advancing field of cell-based therapy: insights and lessons from clinical trials. J Am Heart Assoc 2013;2:e000338

8. Chin SP, Poey AC, Wong CY, et al. Cryopreserved mesenchymal stromal cell treatment is safe and feasible for severe dilated ischemic cardiomyopathy. Cytotherapy 2010;12:31-7.

9. Chin SP, Poey AC, Wong CY, et al. Intramyocardial and intracoronary autologous bone marrow-derived mesenchymal stromal cell treatment in chronic severe dilated cardiomyopathy. Cytotherapy 2011;13:814-21.

10. Wong CY, Chang YM, Tsai YS, et al. Decoding the differentiation of mesenchymal stem cells into mesangial cells at the transcriptomic level. BMC Genomics 2020;21:467.

11. Wong CY, Cheong SK, Mok PL, et al. Differentiation of human mesenchymal stem cells into mesangial cells in post-glomerular injury murine model. Pathology 2008;40:52-7.

12. de Jong R, Houtgraaf JH, Samiei S, et al. Intracoronary stem cell infusion after acute myocardial infarction. Circ Cardiovasc Interv 2014;7:156-67.

13. Holley CT, Long EK, Lindsey ME, et al. Recovery of hibernating myocardium: what is the role of surgical revascularization? J Card Surg 2015;30:224-31.

14. Mingliang R, Bo Z, Zhengguo W. Stem cells for cardiac repair: status, mechanisms, and new strategies. Stem Cells Int 2011;2011:310928.

15. Toma C, Pittenger MF, Cahill KS, et al. Human mesenchymal stem cells differentiate to a cardiomyocyte phenotype in the adult murine heart. Circulation 2002;105:93-8.

16. Pereira WC, Khushnooma I, Madkaikar M, et al. Reproducible methodology for the isolation of mesenchymal stem cells from human umbilical cord and its potential for cardiomyocyte generation. J Tissue Eng Regen Med 2008;2:394-9.

17. Mirotsou M, Jayawardena TM, Schmeckpeper J, at el. Paracrine mechanisms of stem cell reparative and regenerative actions in the heart. J Mol Cell Cardiol 2011;50:280-9.

18. Feng $Y$, Huang $W$, Wani $M$, et al. Ischemic preconditioning potentiates the protective effect of stem cells through secretion of exosomes by targeting Mecp2 via miR-22. PLoS One 2014;9:e88685.

19. Boyle AJ, Whitbourn R, Schlicht S, et al. Intra-coronary high-dose CD34+ stem cells in patients with chronic ischemic heart disease: a 12-month follow-up. Int J Cardiol 2006;109:21-7.

20. Dimmeler S, Burchfield J, Zeiher AM. Cell-based therapy of myocardial infarction. Arterioscler Thromb Vasc Biol 2008;28:208-16.

21. Rota M, Kajstura J, Hosoda T, et al. Bone marrow cells adopt the cardiomyogenic fate in vivo. Proc Natl Acad Sci 2007;104:17783-8.

22. Marmont AM. New horizons in the treatment of 
autoimmune diseases: immunoablation and stem cell transplantation. Annu Rev Med 2000;51:115-34.

23. Weiss ARR, Dahlke MH. Immunomodulation by mesenchymal stem cells (MSCs): mechanisms of action of living, apoptotic, and dead MSCs. Front Immunol 2019;10:1191.

doi: $10.21037 /$ sci-2020-026

Cite this article as: Chin SP, Maskon O, Tan CS, Anderson JE, Wong CY, Hassan HHC, Choor CK, Fadilah SAW, Cheong SK. Synergistic effects of intracoronary infusion of autologous bone marrow-derived mesenchymal stem cells and revascularization procedure on improvement of cardiac function in patients with severe ischemic cardiomyopathy. Stem Cell Investig 2021;8:2. 